\title{
The existence of solutions for a nonlinear mixed problem of singular fractional differential equations
}

\author{
Dumitru Baleanu ${ }^{1,2,3^{*}}$, Hakimeh Mohammadi ${ }^{4}$ and Shahram Rezapour ${ }^{4}$
}

\section{"Correspondence:}

dumitru@cankaya.edu.tr

'Department of Chemical and

Materials Engineering, Faculty of

Engineering, King Abdulaziz

University, P.O. Box 80204, Jeddah,

21589, Saudi Arabia

${ }^{2}$ Department of Mathematics,

Cankaya University, Ogretmenler

Cad. 14, Balgat, Ankara, 06530,

Turkey

Full list of author information is

available at the end of the article

\begin{abstract}
By using fixed point results on cones, we study the existence of solutions for the singular nonlinear fractional boundary value problem

$$
\begin{aligned}
& { }^{c} D^{\alpha} u(t)=f\left(t, u(t), u^{\prime}(t),{ }^{c} D^{\beta} u(t)\right), \\
& u(0)=a u(1), \quad u^{\prime}(0)=b^{c} D^{\beta} u(1), \quad u^{\prime \prime}(0)=u^{\prime \prime \prime}(0)=u^{(n-1)}(0)=0,
\end{aligned}
$$

where $n \geq 3$ is an integer, $\alpha \in(n-1, n), 0<\beta<1,0<a<1,0<b<\Gamma(2-\beta), f$ is an $L^{q}$-Caratheodory function, $q>\frac{1}{\alpha-1}$ and $f(t, x, y, z)$ may be singular at value 0 in one dimension of its space variables $x, y, z$. Here, ${ }^{C} D$ stands for the Caputo fractional derivative.
\end{abstract}

Keywords: boundary value problem; fixed point; fractional differential equation; Green function; regularization; singular

\section{Introduction}

Fractional differential equations (see, for example, [1-6] and references therein) started to play an important role in several branches of science and engineering. There are some works about existence of solutions for the nonlinear mixed problems of singular fractional boundary value problem (see, for example, [7-11] and [12]). Also, there are different methods for solving distinct fractional differential equations (see, for example, [13-18] and [19]). By using fixed point results on cones, we focus on the existence of positive solutions for a nonlinear mixed problem of singular fractional boundary value problem. For the convenience of the reader, we present some necessary definitions from fractional calculus theory (see, for example, [20]). The Caputo derivative of fractional order $\alpha$ for a function $f:[0, \infty) \rightarrow \mathbb{R}$ is defined by

$$
{ }^{c} D^{\alpha} f(t)=\frac{1}{\Gamma(n-\alpha)} \int_{0}^{t}(t-s)^{n-\alpha-1} f^{(n)}(s) d s \quad(n-1<\alpha<n, n=[\alpha]+1) .
$$

Let $q \geq 1$. As you know, $L^{q}[0,1]$ denotes the space of functions, whose $q$ th powers of modulus are integrable on $[0,1]$, equipped with the norm $\|x\|_{q}=\left(\int_{0}^{1}|x(t)|^{q} d t\right)^{\frac{1}{q}}$. We consider the sup norm

$$
\|x\|=\sup \{|x(t)|: t \in[0,1]\}
$$


on the space $C[0,1]$. Also, $A C[0,1]$ is the set of absolutely continuous functions on $[0,1]$. Let $B$ be a subset of $\mathbb{R}^{3}$. A function $f:[0,1] \times B \rightarrow \mathbb{R}$ is called an $L^{q}$-Caratheodory function whenever the real-valued function $f(\cdot, x, y, z)$ on $[0,1]$ is measurable for all $(x, y, z) \in B$, the function $f(t, \cdot, \cdot, \cdot): B \rightarrow \mathbb{R}$ is continuous for almost all $t \in(0,1]$, and for each compact set $U \subset B$, there exists a function $\varphi_{u} \in L^{q}[0,1]$ such that $|f(t, x, y, z)| \leq \varphi_{u}(t)$ for almost all $t \in[0,1]$ and $(x, y, z) \in U$. Consider the nonlinear fractional boundary value problem

$$
\begin{aligned}
& { }^{c} D^{\alpha} u(t)=f\left(t, u(t), u^{\prime}(t),{ }^{c} D^{\beta} u(t)\right), \\
& u(0)=a u(1), \quad u^{\prime}(0)=b^{c} D^{\beta} u(1), \quad u^{\prime \prime}(0)=u^{\prime \prime \prime}(0)=u^{(n-1)}(0)=0,
\end{aligned}
$$

where $n \geq 3$ is an integer, $\alpha \in(n-1, n), 0<\beta<1,0<a<1,0<b<\Gamma(2-\beta)$ and $q>\frac{1}{\alpha-1}$. We say that the function $u:[0,1] \rightarrow \mathbb{R}$ is a positive solution for the problem whenever $u>0$ on $[0,1],{ }^{c} D^{\alpha} u$ is a function in $L^{q}[0,1]$, and $u$ satisfies the boundary conditions almost everywhere on $[0,1]$. In this paper, we suppose that $f$ is an $L^{q}$-Caratheodory function on $[0,1] \times B$, where $B=(0, \infty) \times(0, \infty) \times(0, \infty)$, there exists a positive constant $m$ such that $m \leq f(t, x, y, z)$ for almost all $t \in[0,1]$ and $(x, y, z) \in B, f$ satisfies the estimate

$$
f(t, x, y, z) \leq h(x)+r(|y|)+k(|z|)+\gamma(t) w(x,|y|,|z|),
$$

where $h, r, k \in C(0, \infty)$ are positive and non-increasing, $\gamma \in L^{q}[0,1]$ and $w \in C([0, \infty) \times$ $[0, \infty) \times[0, \infty))$ are positive, $w$ is non-decreasing in all its variables, $\int_{0}^{1} h^{q}\left(s^{\alpha}\right) d s<\infty$, $\int_{0}^{1} r^{q}\left(s^{\alpha-1}\right) d s<\infty, \int_{0}^{1} k^{q}\left(s^{\alpha-\beta}\right) d s<\infty$, and $\lim _{x \mapsto \infty} \frac{w(x, x, x)}{x}=0$. Since we suppose that problem $(*)$ is singular, that is, $f(t, x, y, z)$ may be singular at the value 0 of its space variables $x$, $y, z$, we use regularization and sequential techniques for the existence of positive solutions of the problem. In this way, for each natural number $n$ define the function $f_{n}$ by

$$
f_{n}(t, x, y, z)=f\left(t, \chi_{n}^{+}(x), \chi_{n}^{+}(y), \chi_{n}^{+}(z)\right)
$$

for all $t \in[0,1]$ and $(x, y, z) \in \mathbb{R}^{3}$, where

$$
\chi_{n}^{+}(u)= \begin{cases}u, & u \geq \frac{1}{n}, \\ \frac{1}{n}, & u<\frac{1}{n} .\end{cases}
$$

It is easy to see that each $f_{n}$ is an $L^{q}$-Caratheodory function on $[0,1] \times \mathbb{R}^{3}, m \leq f_{n}(t, x, y, z)$,

$$
f_{n}(t, x, y, z) \leq h\left(\frac{1}{n}\right)+r\left(\frac{1}{n}\right)+k\left(\frac{1}{n}\right)+\gamma(t) w(1+x, 1+|y|, 1+|z|)
$$

and

$$
f_{n}(t, x, y, z) \leq h(x)+r(|y|)+k(|z|)+\gamma(t) w(1+x, 1+|y|, 1+|z|)
$$

for almost all $t \in[0,1]$ and all $(x, y, z) \in B$. In 2012, Agarwal et al. proved the following result. 
Lemma 1.1 [7] Let $\rho \in L^{q}[0,1]$ and $0 \leq t_{1}<t_{2} \leq 1$. Then we have $\left|\int_{0}^{t}(t-s)^{\alpha-2} \rho(s) d s\right| \leq$ $\left(\frac{t^{d}}{d}\right)^{1 / p}\|\rho\|_{q}$ for all $t \in[0,1]$ and

$$
\begin{aligned}
& \left|\int_{0}^{t_{2}}\left(t_{2}-s\right)^{\alpha-2} \rho(s) d s-\int_{0}^{t_{1}}\left(t_{1}-s\right)^{\alpha-2} \rho(s) d s\right| \\
& \quad \leq\left(\frac{t_{1}^{d}+\left(t_{2}-t_{1}\right)^{d}-t_{2}^{d}}{d}\right)^{1 / p}\|\rho\|_{q}+\left(\frac{\left(t_{2}-t_{1}\right)^{d}}{d}\right)^{1 / p}\|\rho\|_{q}
\end{aligned}
$$

where $d=(\alpha-2) p+1$

\section{Main results}

Now, we are ready to investigate the problem in regular and singular cases. First, we give the following result.

Lemma 2.1 Let $y \in C[0,1]$. Then the boundary value problem

$$
\begin{aligned}
& { }^{c} D^{\alpha} u(t)=y(t) \quad(t \in(0,1)), \\
& u(0)=a u(1), \quad u^{\prime}(0)=b^{c} D^{\beta} u(1), \quad u^{\prime \prime}(0)=u^{\prime \prime \prime}(0)=u^{(n-1)}(0)=0
\end{aligned}
$$

is equivalent to the fractional integral equation $u(t)=\int_{0}^{1} G(t, s) y(s) d s$, where

$$
\begin{aligned}
G(t, s)= & \frac{(t-s)^{\alpha-1}}{\Gamma(\alpha)} \\
& +\frac{a \Gamma(\alpha-\beta)(\Gamma(2-\beta)-b)(1-s)^{\alpha-1}+b \Gamma(\alpha) \Gamma(2-\beta)(a+t-a t)(1-s)^{\alpha-\beta-1}}{(1-a) \Gamma(\alpha) \Gamma(\alpha-\beta)(\Gamma(2-\beta)-b)}
\end{aligned}
$$

whenever $0 \leq s \leq t \leq 1$ and

$$
G(t, s)=\frac{a \Gamma(\alpha-\beta)(\Gamma(2-\beta)-b)(1-s)^{\alpha-1}+b \Gamma(\alpha) \Gamma(2-\beta)(a+t-a t)(1-s)^{\alpha-\beta-1}}{(1-a) \Gamma(\alpha) \Gamma(\alpha-\beta)(\Gamma(2-\beta)-b)}
$$

whenever $0 \leq t \leq s \leq 1$.

Proof From ${ }^{c} D^{\alpha} u(t)=y(t)$ and the boundary conditions, we obtain

$$
\begin{aligned}
u(t) & =I^{\alpha} y(t)+u(0)+u^{\prime}(0) t+\frac{u^{\prime \prime}(0)}{2 !} t^{2}+\cdots+\frac{u^{(n-1)}(0)}{(n-1) !} t^{n-1} \\
& =\frac{1}{\Gamma(\alpha)} \int_{0}^{t}(t-s)^{\alpha-1} y(s) d s+u(0)+u^{\prime}(0) t
\end{aligned}
$$

By properties of the Caputo derivative, we get

$$
\begin{aligned}
{ }^{c} D^{\beta} u(t) & =I^{\alpha-\beta} y(t)+{ }^{c} D^{\beta}\left(u(0)+u^{\prime}(0) t\right) \\
& =\frac{1}{\Gamma(\alpha-\beta)} \int_{0}^{t}(t-s)^{\alpha-\beta-1} y(s) d s+\frac{u^{\prime}(0) t^{1-\beta}}{\Gamma(2-\beta)} .
\end{aligned}
$$


Thus, $u(1)=\frac{1}{\Gamma(\alpha)} \int_{0}^{1}(1-s)^{\alpha-1} y(s) d s+u(0)+u^{\prime}(0)$ and

$$
{ }^{c} D^{\beta} u(1)=\frac{1}{\Gamma(\alpha-\beta)} \int_{0}^{1}(1-s)^{\alpha-\beta-1} y(s) d s+\frac{u^{\prime}(0)}{\Gamma(2-\beta)} .
$$

By using the boundary conditions $u(0)=a u(1)$ and $u^{\prime}(0)=b^{c} D^{\beta} u(1)$, we get $u(0)=$ $a\left(\frac{1}{\Gamma(\alpha)} \int_{0}^{1}(1-s)^{\alpha-1} y(s) d s+u(0)+u^{\prime}(0)\right)$ and

$$
u^{\prime}(0)=b\left(\frac{1}{\Gamma(\alpha-\beta)} \int_{0}^{1}(1-s)^{\alpha-\beta-1} y(s) d s+\frac{u^{\prime}(0)}{\Gamma(2-\beta)}\right) .
$$

Hence, $u^{\prime}(0)=\frac{b \Gamma(2-\beta)}{\Gamma(\alpha-\beta)(\Gamma(2-\beta)-b)} \int_{0}^{1}(1-s)^{\alpha-\beta-1} y(s) d s$ and

$$
\begin{aligned}
u(0)= & \frac{a}{(1-a) \Gamma(\alpha)} \int_{0}^{1}(1-s)^{\alpha-1} y(s) d s \\
& +\frac{a b \Gamma(2-\beta)}{(1-a) \Gamma(\alpha-\beta)(\Gamma(2-\beta)-b)} \int_{0}^{1}(1-s)^{\alpha-\beta-1} y(s) d s .
\end{aligned}
$$

Thus,

$$
\begin{aligned}
u(t)= & \frac{1}{\Gamma(\alpha)} \int_{0}^{t}(t-s)^{\alpha-1} y(s) d s+u(0)+u^{\prime}(0) t \\
= & \int_{0}^{t}\left(\frac{(t-s)^{\alpha-1}}{\Gamma(\alpha)}+\frac{a \Gamma(\alpha-\beta)(\Gamma(2-\beta)-b)(1-s)^{\alpha-1}}{(1-a) \Gamma(\alpha) \Gamma(\alpha-\beta)(\Gamma(2-\beta)-b)}\right. \\
& \left.+\frac{b \Gamma(\alpha) \Gamma(2-\beta)(a+t-a t)(1-s)^{\alpha-\beta-1}}{(1-a) \Gamma(\alpha) \Gamma(\alpha-\beta)(\Gamma(2-\beta)-b)}\right) y(s) d s \\
& +\int_{t}^{1}\left(\frac{a \Gamma(\alpha-\beta)(\Gamma(2-\beta)-b)(1-s)^{\alpha-1}}{(1-a) \Gamma(\alpha) \Gamma(\alpha-\beta)(\Gamma(2-\beta)-b)}\right. \\
& \left.+\frac{b \Gamma(\alpha) \Gamma(2-\beta)(a+t-a t)(1-s)^{\alpha-\beta-1}}{(1-a) \Gamma(\alpha) \Gamma(\alpha-\beta)(\Gamma(2-\beta)-b)}\right) y(s) d s \\
= & \int_{0}^{1} G(t, s) y(s) d s .
\end{aligned}
$$

This completes the proof.

Put $k_{1}=\frac{\Gamma(\alpha-\beta)(\Gamma(2-\beta)-b)+b \Gamma(\alpha) \Gamma(2-\beta)}{(1-a) \Gamma(\alpha) \Gamma(\alpha-\beta)(\Gamma(2-\beta)-b)}$ and $k_{2}=\frac{a b \Gamma(2-\beta)}{(1-a) \Gamma(\alpha-\beta)(\Gamma(2-\beta)-b)}$. It is easy to check that the Green function $G$ in the last result belongs to $C([0,1] \times[0,1]), G(t, s)>0$ for all $(t, s) \in$ $[0,1) \times[0,1)$,

$$
G(t, s) \leq k_{1}(1-s)^{\alpha-\beta-1} \leq 1 \quad \text { and } \quad G(t, s) \geq k_{2}(1-s)^{\alpha-\beta-1}
$$

for all $(t, s) \in[0,1] \times[0,1]$. Consider the Banach space $X=C^{1}[0,1]$ with the norm $\|x\|_{*}=$ $\max \left\{\|x\|,\left\|x^{\prime}\right\|\right\}$ and the cone

$$
P=\left\{x \in X: x(t) \geq 0 \text { and } x^{\prime}(t) \geq 0 \text { for all } t \in[0,1]\right\} .
$$


For each natural number $n$, define the operator $Q_{n}$ on $P$ by

$$
\left(Q_{n} x\right)(t)=\int_{0}^{1} G(t, s) f_{n}\left(s, u(s), u^{\prime}(s),{ }^{c} D^{\beta} u(s)\right) d s .
$$

Now, we prove that $Q_{n}$ is a completely continuous operator (see [2]).

Lemma 2.2 The operator $Q_{n}$ is a completely continuous operator.

Proof Let $x \in P$. Then, ${ }^{c} D^{\beta} x \in C[0,1]$ and ${ }^{c} D^{\beta} x \geq 0$. Now, define $\rho(t)=f_{n}\left(t, u(t), u^{\prime}(t)\right.$, $\left.{ }^{c} D^{\beta} u(t)\right)$ for almost all $t \in[0,1]$. Then $\rho \in L^{q}[0,1]$ and $\rho(t) \geq m$ for almost all $t \in[0,1]$. By using the properties of fractional integral $I^{\alpha}$, it is easy to see that $Q_{n} x \in C[0,1],\left(Q_{n} x\right)(t) \geq 0$ and

$$
\left(Q_{n} x\right)^{\prime}(t)=\frac{1}{\Gamma(\alpha-1)} \int_{0}^{t}(t-s)^{\alpha-2} \rho(s) d s
$$

for all $t \in[0,1]$. This implies that $\left(Q_{n} x\right)^{\prime} \in C[0,1]$ and $\left(Q_{n} x\right)^{\prime} \geq 0$ on $[0,1]$. Consequently, $Q_{n}$ maps $P$ into $P$. In order to prove that $Q_{n}$ is a continuous operator, let $x_{m}$ be a convergent sequence in $P$ and $\lim _{m \mapsto \infty} x_{m}=x$. Thus, $\lim _{m \mapsto \infty} x_{m}^{(j)}(t)=x^{(j)}(t)$ uniformly on [0,1] for $j=0,1$. Since

$$
\begin{aligned}
{ }^{c} D^{\beta} x(t) & =\frac{1}{\Gamma(1-\beta)} \frac{d}{d t} \int_{0}^{t}(t-s)^{-\beta}(x(s)-x(0)) d s \\
& =\frac{1}{\Gamma(1-\beta)} \int_{0}^{t}(t-s)^{-\beta} x^{\prime}(s) d s,
\end{aligned}
$$

we get $\left|{ }^{c} D^{\beta} x_{m}(t)-{ }^{c} D^{\beta} x(t)\right| \leq \frac{\left\|x_{m}^{\prime}-x^{\prime}\right\|}{\Gamma(1-\beta)} \int_{0}^{t}(t-s)^{-\beta} d s \leq \frac{\left\|x_{m}-x\right\|_{*}}{\Gamma(\beta)}$ and $\lim _{m \mapsto \infty}{ }^{c} D^{\beta} x_{m}(t)=$ ${ }^{c} D^{\beta} x(t)$ uniformly on $[0,1]$. Also, we have $\left|{ }^{c} D^{\beta} x_{m}(t)\right| \leq \frac{x_{m}^{\prime}}{\Gamma(\beta)}$ on $[0,1]$, and so $\left\|{ }^{c} D^{\beta} x_{m}\right\| \leq$ $\frac{\left\|x_{m}^{\prime}\right\|}{\Gamma(\beta)}$. Now, put

$$
\rho_{m}(t)=f_{n}\left(t, x_{m}(t), x_{m}^{\prime}(t),{ }^{c} D^{\beta} x_{m}(t)\right) \quad \text { and } \quad \rho(t)=f_{n}\left(t, x(t), x^{\prime}(t),{ }^{c} D^{\beta} x(t)\right) .
$$

Then, it is easy to see that $\lim _{m \mapsto \infty} \rho_{m}(t)=\rho(t)$ for almost all $t \in[0,1]$, and there exists $\beta \in L^{q}[0,1]$ such that $0 \leq \rho_{m}(t) \leq \beta(t)$ for almost all $t \in[0,1]$ and all $m \geq 1$. Since $f_{n}$ is an $L^{q}$-Caratheodory function, $\left\{x_{m}\right\}$ is bounded in $C^{1}[0,1]$, and $\left\{{ }^{c} D^{\beta} x_{m}\right\}$ is bounded in $C[0,1]$. Therefore, $\lim _{m \mapsto \infty}\left(Q_{n} x_{m}\right)(t)=\left(Q_{n} x\right)(t)$ uniformly on $[0,1]$. Since $\left\{\rho_{m}\right\}$ is $L^{q}$-convergent on $[0,1]$,

$$
\lim _{m \mapsto \infty}\left(Q_{n} x_{m}\right)^{\prime}(t)=\frac{1}{\Gamma(\alpha-1)} \lim _{m \mapsto \infty} \int_{0}^{t}(t-s)^{\alpha-2} \rho_{m}(s) d s=\left(Q_{n} x\right)^{\prime}(t)
$$

uniformly on $[0,1]$. Hence, $Q_{n}$ is a continuous operator. Now, we have to show that for each bounded sequence $\left\{x_{m}\right\}$ in $P$, the sequence $\left\{Q_{n} x_{m}\right\}$ is relatively compact in $C[0,1]$. Choose a positive constant $k$ such that $\left\|x_{m}\right\| \leq k$ and $\left\|x_{m}^{\prime}\right\| \leq k$ for all $m$. Note that $\left\|^{c} D^{\beta} x_{m}\right\| \leq$ $\frac{k}{\Gamma(\beta)}$ and $\left|\int_{0}^{t}(t-s)^{\alpha-2} \rho_{m}(s) d s\right| \leq\left(\int_{0}^{t}(t-s)^{(\alpha-2) p} d s\right)^{\frac{1}{p}}\left(\int_{0}^{t}\left|\rho_{m}(s)\right|^{q} d s\right)^{\frac{1}{q}} \leq\left(\frac{t^{d}}{d}\right)^{\frac{1}{p}}\left\|\rho_{m}\right\|_{q}$ for all $m$, where $d=(\alpha-2) p+1$. But we have

$$
0 \leq\left(Q_{n} x_{m}\right)(t)=\int_{0}^{1} G(t, s) \rho_{m}(s) d s \leq \int_{0}^{1} G(t, s) \beta(s) d s \leq \frac{\|\beta\|_{1}}{\Gamma(\alpha)}
$$


and

$$
\begin{aligned}
0 & \leq\left(Q_{n} x_{m}\right)^{\prime}(t)=\frac{1}{\Gamma(\alpha-1)} \int_{0}^{t}(t-s)^{\alpha-2} \rho_{m}(s) d s \\
& \leq \frac{1}{\Gamma(\alpha-1)} \int_{0}^{t}(t-s)^{\alpha-2} \beta(s) d s \leq \frac{1}{\Gamma(\alpha-1)}\left(\frac{1}{(\alpha-2) p+1}\right)^{\frac{1}{p}}\|\beta\|_{q}
\end{aligned}
$$

for all $t \in[0,1]$ and $m$. This implies that $\left\{Q_{n} x_{m}\right\}$ is bounded in $C^{1}[0,1]$. Also, we have

$$
\begin{aligned}
& \left|\left(Q_{n} x_{m}\right)^{\prime}\left(t_{2}\right)-\left(Q_{n} x_{m}\right)^{\prime}\left(t_{1}\right)\right| \\
& \quad=\frac{1}{\Gamma(\alpha-1)}\left|\int_{0}^{t_{2}}\left(t_{2}-s\right)^{\alpha-2} \rho_{m}(s) d s-\int_{0}^{t_{1}}\left(t_{1}-s\right)^{\alpha-2} \rho_{m}(s) d s\right| \\
& \quad \leq \frac{\left\|\rho_{m}\right\|_{q}}{\Gamma(\alpha-1)}\left(\left(\frac{t_{1}^{d}+\left(t_{2}-t_{1}\right)^{d}-t_{2}^{d}}{d}\right)^{\frac{1}{p}}+\left(\frac{\left(t_{2}-t_{1}\right)^{d}}{d}\right)^{\frac{1}{p}}\right) \\
& \quad \leq \frac{\|\beta\|_{q}}{\Gamma(\alpha-1)}\left(\left(\frac{t_{1}^{d}+\left(t_{2}-t_{1}\right)^{d}-t_{2}^{d}}{d}\right)^{\frac{1}{p}}+\left(\frac{\left(t_{2}-t_{1}\right)^{d}}{d}\right)^{\frac{1}{p}}\right)
\end{aligned}
$$

for all $0 \leq t_{1} \leq t_{2} \leq 1$, where $d=(\alpha-2) p+1$. Hence, $\left\{\left(Q_{n} x_{m}\right)^{\prime}\right\}$ is equicontinuous on $[0,1]$. Thus, $\left\{Q_{n} x_{m}\right\}$ is relatively compact in $C^{1}[0,1]$ by the Arzela-Ascoli theorem. Hence, $Q_{n}$ is a completely continuous operator.

We need the following result (see [2] and [21]).

Lemma 2.3 [21] Let $Y$ be a Banach space, $P$ a cone in $Y$ and $\Omega_{1}$ and $\Omega_{2}$ bounded open balls in $Y$ centered at the origin with $\bar{\Omega}_{1} \subset \Omega_{2}$. Suppose that $T: P \cap\left(\bar{\Omega}_{2} \backslash \Omega_{1}\right) \rightarrow P$ is a completely continuous operator such that $\|T x\| \geq\|x\|$ for all $x \in P \cap \partial \Omega_{1}$ and $\|T x\| \leq\|x\|$ for all $x \in P \cap \partial \Omega_{2}$. Then $T$ has a fixed point in $P \cap\left(\bar{\Omega}_{2} \backslash \Omega_{1}\right)$.

Theorem 2.4 For each natural number $n$, problem $(*)$ has a solution $u_{n} \in P$ such that $u_{n} \geq \frac{m k_{2}}{\alpha-\beta}, u_{n}^{\prime}(t) \geq \frac{m t^{\alpha-1}}{\Gamma(\alpha)}$ and ${ }^{c} D^{\beta} u_{n}(t) \geq \frac{m t^{\alpha-\beta}}{\Gamma(\alpha-\beta+1)}$ for all $t \in[0,1]$.

Proof Let $n \geq 1$. It is sufficient to show that $Q_{n}$ has a fixed point $u_{n}$ in $P$ with the desired conditions. In this way, note that

$$
\begin{aligned}
\left(Q_{n} x\right)(t) & =\int_{0}^{1} G(t, s) f_{n}\left(s, x(s), x^{\prime}(s),{ }^{c} D^{\beta} x(s)\right) d s \\
& \geq m \int_{0}^{1} G(t, s) d s \geq m \int_{0}^{1} k_{2}(1-s)^{\alpha-\beta-1} d s=\frac{m k_{2}}{\alpha-\beta},
\end{aligned}
$$

and so $\left\|Q_{n} x\right\|_{*} \geq\left\|Q_{n}(x)\right\| \geq \frac{m k_{2}}{\alpha-\beta}$. Put $\Omega_{1}=\left\{x \in X:\|x\|_{*}<\frac{m k_{2}}{\alpha-\beta}\right\}$. Then $\left\|Q_{n} x\right\|_{*} \geq\|x\|_{*}$ for all $x \in P \cap \partial \Omega_{1}$. If $v_{n}=h\left(\frac{1}{n}\right)+r\left(\frac{1}{n}\right)+k\left(\frac{1}{n}\right)$, then

$$
\begin{aligned}
\left|\left(Q_{n} x\right)(t)\right| & \leq\left|\int_{0}^{1} G(t, s) f_{n}\left(s, u(s), u^{\prime}(s),{ }^{c} D^{\beta} u(s)\right) d s\right| \\
& \leq \int_{0}^{1}|G(t, s)|\left(v_{n}+\gamma(s) w\left(1+|x(s)|, 1+\left|x^{\prime}(s)\right|, 1+\left|{ }^{c} D^{\beta} x(s)\right|\right)\right) d s \\
& \leq k_{1}\left(v_{n}+w\left(1+\|x\|, 1+\left\|x^{\prime}\right\|, 1+\left\|{ }^{c} D^{\beta} x\right\|\right)\|\gamma\|_{1}\right)
\end{aligned}
$$


and

$$
\begin{aligned}
& \left|\left(Q_{n} x\right)^{\prime}(t)\right| \\
& \quad=\left|\frac{1}{\Gamma(\alpha-1)} \int_{0}^{t}(t-s)^{\alpha-2} f_{n}\left(s, x(s), x^{\prime}(s),{ }^{c} D^{\beta} x(s)\right) d s\right| \\
& \quad \leq \frac{1}{\Gamma(\alpha-1)} \int_{0}^{t}(t-s)^{\alpha-2}\left(v_{n}+\gamma(s) w\left(1+|x(s)|, 1+\left|x^{\prime}(s)\right|, 1+\left|{ }^{c} D^{\beta} x(s)\right|\right)\right) d s \\
& \quad \leq \frac{1}{\Gamma(\alpha-1)}\left(\frac{v_{n} t^{\alpha-1}}{\alpha-1}+w\left(1+\|x\|, 1+\left\|x^{\prime}\right\|, 1+\left\|{ }^{c} D^{\beta} x\right\|\right) \int_{0}^{t}(t-s)^{\alpha-2} \gamma(s) d s\right)
\end{aligned}
$$

for all $x \in P$ and $t \in[0,1]$, because $w$ is non-decreasing in all its variables. Since $\|x\| \leq\|x\|_{*}$, $\left\|x^{\prime}\right\| \leq\|x\|_{*},\left\|{ }^{c} D^{\beta} x\right\| \leq \frac{\left\|x^{\prime}\right\|}{\Gamma(\beta)} \leq \frac{\|x\|_{*}}{\Gamma(\beta)}$ and

$$
\int_{0}^{t}(t-s)^{\alpha-2} \gamma(s) d s \leq\left(\frac{1}{d}\right)^{1 / p}\|\gamma\|_{q}
$$

where $d=(\alpha-2) p+1$, we have

$$
\left\|Q_{n}(x)\right\| \leq k_{1}\left(v_{n}+w\left(1+\|x\|_{*}, 1+\|x\|_{*}, 1+\frac{\|x\|_{*}}{\Gamma(\beta)}\right)\|\gamma\|_{1}\right)
$$

and

$$
\begin{aligned}
& \left\|\left(Q_{n} x\right)^{\prime}\right\| \\
& \quad \leq \frac{1}{\Gamma(\alpha-1)}\left(\frac{v_{n}}{\alpha-1}+w\left(1+\|x\|_{*}, 1+\|x\|_{*}, 1+\frac{\|x\|_{*}}{\Gamma(\beta)}\right)(1 / d)^{1 / p}\|\gamma\|_{q}\right) .
\end{aligned}
$$

Hence, $\left\|Q_{n} x\right\|_{*} \leq M\left(\frac{v_{n}}{\alpha-1}+N w\left(1+\|x\|_{*}, 1+\|x\|_{*}, 1+\frac{\|x\|_{*}}{\Gamma(\beta)}\right)\right)$, where $N=\max \left\{\|\gamma\|_{1},(1 / d)^{1 / p} \times\right.$ $\left.\|\gamma\|_{q}\right\}$ and $M=\max \left\{\frac{1}{\Gamma(\alpha-1)}, k_{1}\right\}$. Since

$$
\lim _{v \mapsto \infty} \frac{1}{v} w(1+v, 1+v, 1+v)=0
$$

there exists a positive constant $L$ such that

$$
M\left(\frac{v_{n}}{\alpha-1}+N w\left(1+v, 1+v, \frac{v}{\Gamma(\beta)}\right)\right)<v
$$

for all $v \geq L$. Thus, $\left\|Q_{n} x\right\|_{*}<\|x\|_{*}$ for all $x \in P$ with $\|x\|_{*} \geq L$. Put $\Omega_{2}=\left\{x \in X:\|x\|_{*}<L\right\}$. Then $\left\|Q_{n} x\right\|_{*}<\|x\|_{*}$ for all $x \in P \cap \partial \Omega_{2}$. By using last result, $Q_{n}$ has a fixed point $u_{n}$ in $P \cap\left(\bar{\Omega}_{2} \backslash \Omega_{1}\right)$. But $u_{n}=\left(Q_{n} u_{n}\right)(t) \geq \frac{m k_{2}}{\alpha-\beta}$ and

$$
\begin{aligned}
\left(Q_{n} x\right)^{\prime}(t) & =\frac{1}{\Gamma(\alpha-1)} \int_{0}^{t}(t-s)^{\alpha-2} f_{n}\left(s, x(s), x^{\prime}(s),{ }^{c} D^{\beta} x(s)\right) d s \\
& \geq \frac{m}{\Gamma(\alpha-1)} \int_{0}^{t}(t-s)^{\alpha-2} d s=\frac{m t^{\alpha-1}}{\Gamma(\alpha)}
\end{aligned}
$$


for all $t \in[0,1]$ and $x \in P$. Since $\int_{0}^{t}(t-s)^{-\beta} s^{\alpha-1} d s=\frac{\Gamma(\alpha) \Gamma(1-\beta)}{\Gamma(\alpha-\beta+1)} t^{\alpha-\beta}$,

$$
\begin{aligned}
{ }^{c} D^{\beta} u_{n}(t) & =\frac{1}{\Gamma(1-\beta)} \int_{0}^{t}(t-s)^{-\beta} u_{n}^{\prime}(s) d s \\
& \geq \frac{m}{\Gamma(\alpha) \Gamma(1-\beta)} \int_{0}^{t}(t-s)^{-\beta} s^{\alpha-1} d s=\frac{m t^{\alpha-\beta}}{\Gamma(\alpha-\beta+1)}
\end{aligned}
$$

for all $t \in[0,1]$. This completes the proof.

Now, we give our last result.

Theorem 2.5 Problem (*) has a solution $u$ such that $u(t) \geq \frac{m k_{2}}{\alpha-\beta}, u^{\prime}(t) \geq \frac{m t^{\alpha-1}}{\Gamma(\alpha)}$ and ${ }^{c} D^{\beta} u(t) \geq \frac{m t^{\alpha-\beta}}{\Gamma(\alpha-\beta+1)}$ for all $t \in[0,1]$.

Proof By using Theorem 2.4, one gets that for each natural number $n$, problem $(*)$ has a solution $u_{n} \in P$ with the desired conditions. Thus, $h\left(u_{n}(t)\right) \leq h\left(\frac{m k_{2}}{\alpha-\beta}\right), r\left(\left|u_{n}^{\prime}(t)\right|\right) \leq r\left(\frac{m t^{\alpha-1}}{\Gamma(\alpha)}\right)$ and $k\left(\left|{ }^{c} D^{\beta} u_{n}(t)\right|\right) \leq k\left(\frac{m t^{\alpha-\beta}}{\Gamma(\alpha-\beta+1)}\right)$ for all $t \in[0,1]$ and $n$. Also, we have $\left\|^{c} D^{\beta} u_{n}\right\| \leq \frac{\left\|u_{n}^{\prime}\right\|}{\Gamma(\beta)}$. Suppose that

$$
S(t)=h\left(\frac{m k_{2}}{\alpha-\beta}\right)+r\left(\frac{m t^{\alpha-1}}{\Gamma(\alpha)}\right)+k\left(\frac{m t^{\alpha-\beta}}{\Gamma(\alpha-\beta+1)}\right) .
$$

Then

$$
\begin{aligned}
m & \leq f_{n}\left(t, u_{n}(t), u_{n}^{\prime}(t),{ }^{c} D^{\beta} u_{n}(t)\right) \\
& \leq S(t)+\gamma(t) w\left(1+\left\|u_{n}\right\|, 1+\left\|u_{n}^{\prime}\right\|, 1+\left\|{ }^{c} D^{\beta} u_{n}\right\|\right) \\
& \leq S(t)+\gamma(t) w\left(1+\left\|u_{n}\right\|_{*}, 1+\left\|u_{n}^{\prime}\right\|_{*}, 1+\frac{\left\|u_{n}\right\|_{*}}{\Gamma(\beta)}\right)
\end{aligned}
$$

for almost all $t \in[0,1]$ and $n$. Since $0 \leq G(t, s) \leq k_{1}$, we get

$$
\begin{aligned}
0 & \leq u_{n}(t) \\
& =\int_{0}^{1} G(t, s) f_{n}\left(s, u_{n}(s), u_{n}^{\prime}(s),{ }^{c} D^{\beta} u_{n}(s)\right) d s \\
& \leq k_{1}\left(\int_{0}^{1} S(s) d s+w\left(1+\left\|u_{n}\right\|_{*}, 1+\left\|u_{n}\right\|_{*}, 1+\frac{\left\|u_{n}\right\|_{*}}{\Gamma(\beta)}\right)\|\gamma\|_{1}\right)
\end{aligned}
$$

and

$$
\begin{aligned}
0 \leq & u_{n}^{\prime}(t) \\
\leq & \frac{1}{\Gamma(\alpha-1)}\left(\int_{0}^{t}(t-s)^{\alpha-2} S(s) d s\right. \\
& \left.+w\left(1+\left\|u_{n}\right\|_{*}, 1+\left\|u_{n}\right\|_{*}, 1+\frac{\left\|u_{n}\right\|_{*}}{\Gamma(\beta)}\right) \int_{0}^{t}(t-s)^{\alpha-2} \gamma(s) d s\right) .
\end{aligned}
$$


We show that $\int_{0}^{t}(t-s)^{\alpha-2} S(s) d s$ is bounded on $[0,1]$. Let $d=(\alpha-2) p+1$. Note that

$$
\begin{aligned}
& \int_{0}^{1}(t-s)^{\alpha-2} h\left(\frac{m k_{2}}{\alpha-\beta}\right) d s \\
& \quad=h\left(\frac{m k_{2}}{\alpha-\beta}\right) \int_{0}^{1}(t-s)^{\alpha-2} d s=\frac{1}{\alpha-1} h\left(\frac{m k_{2}}{\alpha-\beta}\right)=: \eta_{1}<\infty, \\
& \int_{0}^{t}(t-s)^{\alpha-2} r\left(\frac{m s^{\alpha-1}}{\Gamma(\alpha)}\right) d s \\
& =\left(\frac{1}{d}\right)^{1 / p}\left(\frac{\Gamma(\alpha)}{m}\right)^{\frac{1}{(\alpha-1) q}}\left(\int_{0}^{\left(\frac{m}{\Gamma(\alpha)}\right)^{\frac{1}{\alpha-1}}} r^{q}\left(s^{\alpha-1}\right) d s\right)^{1 / q}=: \eta_{2}<\infty
\end{aligned}
$$

and

$$
\begin{aligned}
& \int_{0}^{t}(t-s)^{\alpha-2} k\left(\frac{m s^{\alpha-\beta}}{\Gamma(\alpha-\beta+1)}\right) d s \\
& \quad=\left(\frac{1}{d}\right)^{1 / p}\left(\frac{\Gamma(\alpha-\beta+1)}{m}\right)^{\frac{1}{(\alpha-\beta) q}}\left(\int_{0}^{\left(\frac{m}{\Gamma(\alpha-\beta+1)}\right)^{\frac{1}{\alpha-\beta}}} k^{q}\left(s^{\alpha-\beta}\right) d s\right)^{1 / q}=: \eta_{3}<\infty .
\end{aligned}
$$

Thus, $\int_{0}^{t}(t-s)^{\alpha-2} S(s) d s \leq \eta$ for all $t \in[0,1]$, where $\eta=\eta_{1}+\eta_{2}+\eta_{3}$. Also, we have

$$
\begin{aligned}
& \int_{0}^{1} S(s) d s \\
& \quad \leq \frac{1}{\alpha-1} h\left(\frac{m k_{2}}{\alpha-\beta}\right)+\left(\frac{\Gamma(\alpha)}{m}\right)^{\frac{1}{\alpha-1}} \int_{0}^{\left(\frac{m}{\Gamma(\alpha)}\right)^{\frac{1}{\alpha-1}}} r\left(s^{\alpha-1}\right) d s \\
& \quad+\left(\frac{\Gamma(\alpha-\beta+1)}{m}\right)^{\frac{1}{\alpha-\beta}} \int_{0}^{\left(\frac{m}{\Gamma(\alpha-\beta+1)}\right)^{\frac{1}{\alpha-\beta}} k\left(s^{\alpha-\beta}\right) d s<\infty .}
\end{aligned}
$$

Since

$$
\left\|u_{n}\right\|=k_{1}\left(\int_{0}^{1} S(s) d s+w\left(1+\left\|u_{n}\right\|_{*}, 1+\left\|u_{n}\right\|_{*}, 1+\frac{\left\|u_{n}\right\|_{*}}{\Gamma(\beta)}\right)\|\gamma\|_{1}\right)
$$

and

$$
\left\|u_{n}^{\prime}\right\| \leq \frac{1}{\Gamma(\alpha-1)}\left(\eta+w\left(1+\left\|u_{n}\right\|_{*}, 1+\left\|u_{n}\right\|_{*}, 1+\frac{\left\|u_{n}\right\|_{*}}{\Gamma(\beta)}\right)\left(\frac{1}{d}\right)^{1 / p}\|\gamma\|_{q}\right)
$$

we get $\left\|u_{n}\right\|_{*} \leq M\left(\Phi+K w\left(1+\left\|u_{n}\right\|_{*}, 1+\left\|u_{n}\right\|_{*}, 1+\frac{\left\|u_{n}\right\|_{*}}{\Gamma(\beta)}\right)\right)$ for all $n$, where $\Phi=\max \{\eta$, $\left.\int_{0}^{1} S(s) d s\right\}, K=\max \left\{\|\gamma\|_{1},\left(\frac{1}{d}\right)^{1 / p}\|\gamma\|_{q}\right\}$ and also $M=\max \left\{k_{1}, \frac{1}{\Gamma(\alpha-1)}\right\}$. On the other hand, there exists a positive constant $L$ such that $M\left(\Phi+K w\left(1+v, 1+v, 1+\frac{v}{\Gamma(\beta)}\right)\right)<v$ for all $v \geq L$, and so $\left\|u_{n}\right\|_{*}<L$ for all $n$. Thus, for almost all $t \in[0,1]$ and all $n$, we have $f_{n}\left(t, u_{n}(t), u_{n}^{\prime}(t),{ }^{c} D^{\beta} u_{n}(t)\right) \leq R(t)$, where

$$
R(t)=S(t)+\gamma(t) w\left(1+L, 1+L, 1+\frac{L}{\Gamma(\beta)}\right)
$$


Note that $R \in L^{q}[0,1]$. We show that $\left\{u_{n}^{\prime}\right\}$ is equicontinuous on $[0,1]$. Let $\rho_{n}(t)=$ $f_{n}\left(t, u_{n}(t), u_{n}^{\prime}(t),{ }^{c} D^{\beta} u_{n}(t)\right)$ and $0 \leq t_{1}<t_{2} \leq T$. Then

$$
\begin{aligned}
& \left|u_{n}^{\prime}\left(t_{2}\right)-u_{n}^{\prime}\left(t_{1}\right)\right| \\
& \quad=\frac{1}{\Gamma(\alpha-1)}\left|\int_{0}^{t_{2}}\left(t_{2}-s\right)^{\alpha-2} \rho_{n}(s) d s-\int_{0}^{t_{1}}\left(t_{1}-s\right)^{\alpha-2} \rho_{n}(s) d s\right| \\
& \quad \leq \frac{1}{\Gamma(\alpha-1)}\left(\int_{0}^{t_{1}}\left(\left(t_{1}-s\right)^{\alpha-2}-\left(t_{2}-s\right)^{\alpha-2}\right) \rho_{n}(s) d s+\int_{t_{1}}^{t_{2}}\left(t_{2}-s\right)^{\alpha-2} \rho_{n}(s) d s\right) \\
& \quad \leq \frac{1}{\Gamma(\alpha-1)}\left(\int_{0}^{t_{1}}\left(\left(t_{1}-s\right)^{\alpha-2}-\left(t_{2}-s\right)^{\alpha-2}\right) R(s) d s+\int_{t_{1}}^{t_{2}}\left(t_{2}-s\right)^{\alpha-2} R(s) d s\right),
\end{aligned}
$$

and so

$$
\begin{aligned}
& \left|u_{n}^{\prime}\left(t_{2}\right)-u_{n}^{\prime}\left(t_{1}\right)\right| \\
& \quad \leq \frac{\|R\|_{q}}{\Gamma(\alpha-1)}\left(\left(\frac{t_{1}^{d}+\left(t_{2}-t_{1}\right)^{d}-t_{2}^{d}}{d}\right)^{1 / p}+\left(\frac{\left(t_{2}-t_{1}\right)^{d}}{d}\right)^{1 / p}\right) .
\end{aligned}
$$

Hence, $\left\{u_{n}^{\prime}\right\}$ is equicontinuous on $[0,1]$. Since $\left\{u_{n}\right\}$ is a bounded sequence in $C[0,1]$, by using the Arzela-Ascoli theorem, without loss of generality, we can assume that $\left\{u_{n}\right\}$ is convergent in $C[0,1]$. Let $\lim _{n \mapsto \infty} u_{n}=u$. Then, it is easy to see that ${ }^{c} D^{\beta} u_{n}(t)=\frac{1}{\Gamma(\alpha-1)} \int_{0}^{t}(t-$ $s)^{-\beta} u_{n}^{\prime}(s) d s$, and ${ }^{c} D^{\beta} u_{n}(t)$ uniformly converges to $\frac{1}{\Gamma(\alpha-1)} \int_{0}^{t}(t-s)^{-\beta} u^{\prime}(s) d s$ on $[0,1]$. Thus, ${ }^{c} D^{\beta} u_{n}$ converges to ${ }^{c} D^{\beta} u$ in $C[0,1]$. Hence,

$$
\lim _{n \mapsto \infty} f_{n}\left(t, u_{n}(t), u_{n}^{\prime}(t),{ }^{c} D^{\beta} u_{n}(t)\right)=f\left(t, u(t), u^{\prime}(t),{ }^{c} D^{\beta} u(t)\right)
$$

for almost all $t \in[0,1]$. Since $R \in L^{q}[0,1]$, by using the dominated convergence theorem on the relation

$$
u_{n}(t)=\int_{0}^{1} G(t, s) f_{n}\left(s, u_{n}(s), u_{n}^{\prime}(s),{ }^{c} D^{\beta} u_{n}(s)\right) d s,
$$

we get $u(t)=\int_{0}^{1} G(t, s) f\left(s, u(s), u^{\prime}(s),{ }^{c} D^{\beta} u(s)\right)$ for all $t \in[0,1]$. This completes the proof.

\subsection{Examples for the problem}

Example 2.1 Let $\rho_{1}, \rho_{2} \in L^{q}[0,1], \rho_{1}(t) \geq m>0$ for almost all $t$ in $[0,1]$. Suppose that

$$
f(t, x, y, z)=\rho_{1}(t)+\frac{1}{x^{\frac{1}{3}}-\lambda}+\frac{1}{y^{\frac{1}{4}}}+\frac{1}{z^{\frac{1}{4}}}+\left|\rho_{2}(t)\right|\left(x^{\frac{1}{3}}+y^{\frac{1}{4}}+z^{\frac{1}{4}}\right)
$$

on $[0,1] \times B, \lambda=(a u(1))^{\frac{1}{3}}, h(x)=\frac{1}{x^{\frac{1}{3}}-\lambda}$ whenever $x^{\frac{1}{3}}-\lambda \geq 0$ and $h(x)=0$ whenever $x^{\frac{1}{3}}-$ $\lambda<0, r(x)=\frac{1}{x^{\frac{1}{4}}}, k(x)=\frac{1}{x^{\frac{1}{4}}}, w(x, y, z)=\left(x^{\frac{1}{3}}+y^{\frac{1}{4}}+z^{\frac{1}{4}}+1\right)$ and $\gamma(t)=\rho_{1}(t)+\left|\rho_{2}(t)\right|$. Then Theorem 2.5 guarantees that problem $(*)$ has a positive solution. 
Example 2.2 Consider the nonlinear mixed problem of singular fractional boundary value problem

$$
\begin{aligned}
{ }^{c} D^{\frac{7}{3}} u(t)= & t+1+\frac{1}{u(t)^{\frac{1}{3}}-\rho}+\frac{1}{u^{\prime}(t)^{\frac{1}{4}}}+\frac{1}{\left({ }^{c} D^{\frac{1}{4}} u(t)\right)^{\frac{1}{4}}} \\
& +2\left(u(t)^{\frac{1}{3}}+u^{\prime}(t)^{\frac{1}{4}}+\left({ }^{c} D^{\frac{1}{4}} u(t)\right)^{\frac{1}{4}}+1\right)
\end{aligned}
$$

via boundary value conditions $u(0)=\frac{1}{4} u(1), u^{\prime}(0)=\frac{1}{3}\left({ }^{c} D^{\frac{1}{4}}\right) u(1)$ and $u^{\prime \prime}(0)=u^{\prime \prime \prime}(0)=\cdots=$ $u^{(n-1)}(0)=0$, where $\rho=\left(\left(\frac{1}{4}\right) u(1)\right)^{\frac{1}{3}}$. Let

$$
\begin{aligned}
f\left(t, u(t), u^{\prime}(t),{ }^{c} D^{\frac{1}{4}}\right)= & t+1+\frac{1}{u(t)^{\frac{1}{3}}-\rho}+\frac{1}{u^{\prime}(t)^{\frac{1}{4}}}+\frac{1}{\left({ }^{c} D^{\frac{1}{4}} u(t)\right)^{\frac{1}{4}}} \\
& +2\left(u(t)^{\frac{1}{3}}+u^{\prime}(t)^{\frac{1}{4}}+\left({ }^{c} D^{\frac{1}{4}} u(t)\right)^{\frac{1}{4}}+1\right) .
\end{aligned}
$$

Then the map $f$ is singular at $t=0$, and $f$ satisfies the desired conditions, where $h(x)=\frac{1}{x^{\frac{1}{3}}-\rho}$ whenever $x^{\frac{1}{3}}-\rho \geq 0$ and $h(x)=0$ whenever $x^{\frac{1}{3}}-\rho<0, r(x)=\frac{1}{x^{\frac{1}{4}}}, k(x)=\frac{1}{x^{\frac{1}{4}}}, w(x, y, z)=$ $x^{\frac{1}{3}}+y^{\frac{1}{4}}+z^{\frac{1}{4}}+1, \rho_{1}(t)=t+1>1=m, \rho_{2}(t)=2$ and $\gamma(t)=\rho_{1}(t)+\left|\rho_{2}(t)\right|$. Then Theorem 2.5 guarantees that this problem has a positive solution.

\section{Conclusions}

One of the most interesting branches is obtaining solutions of singular fractional differential via boundary value problems. Having these things in mind, we study the existence of solutions for a singular nonlinear fractional boundary value problem. Two illustrative examples illustrate the applicability of the proposed method. It seems that the obtained results could be extended to more general functional spaces. Finally, note that all calculations in proofs of the results depend on the definition of the fractional derivative.

Competing interests

The authors declare that they have no competing interests.

Authors' contributions

All authors have equal contributions. All authors read and approved the final manuscript.

\section{Author details}

1 Department of Chemical and Materials Engineering, Faculty of Engineering, King Abdulaziz University, P.O. Box 80204, Jeddah, 21589, Saudi Arabia. ${ }^{2}$ Department of Mathematics, Cankaya University, Ogretmenler Cad. 14, Balgat, Ankara, 06530, Turkey. ${ }^{3}$ Institute of Space Sciences, Magurele, Bucharest, Romania. ${ }^{4}$ Department of Mathematics, Azarbaijan Shahid Madani University, Azarshahr, Tabriz, Iran

\section{Acknowledgements}

Research of the second and third authors was supported by Azarbaijan Shahid Madani University. Also, the authors express their gratitude to the referees for their helpful suggestions, which improved the final version of this paper.

Received: 27 May 2013 Accepted: 28 August 2013 Published: 11 Dec 2013

\section{References}

1. Bai, Z, Lu, H: Positive solutions for boundary value problem of nonlinear fractional differential equation. J. Math. Anal. Appl. 311, 495-505 (2005)

2. Baleanu, D, Mohammadi, H, Rezapour, S: Positive solutions of a boundary value problem for nonlinear fractional differential equations. Abstr. Appl. Anal. 2012, Article ID 837437 (2012)

3. Baleanu, D, Mohammadi, H, Rezapour, S: Some existence results on nonlinear fractional differential equations. Philos. Trans. R. Soc. Lond. A 371, 20120144 (2013)

4. Baleanu, D, Mohammadi, $H$, Rezapour, S: On a nonlinear fractional differential equation on partially ordered metric spaces. Adv. Differ. Equ. 2013, 83 (2013) 
5. Kilbas, AA, Trujillo, JJ: Differential equations of fractional order: methods, results and problems. Appl. Anal. 81(2), 435-493 (2002)

6. Zhang, SQ: Existence of positive solution for some class of nonlinear fractional differential equations. J. Math. Anal. Appl. 278, 136-148 (2003)

7. Agarwal, RP, O'Regan, D, Stanek, S: Positive solutions for mixed problems of singular fractional differential equations. Math. Nachr. 285(1), 27-41 (2012)

8. Cabrera, IJ, Harjani, J, Sadarangani, KB: Existence and uniqueness of positive solutions for a singular fractional three-point boundary value problem. Abstr. Appl. Anal. 2012, Article ID 803417 (2012)

9. Cao, J, Chen, H: Positive solution of singular fractional differential equation in Banach space. J. Appl. Math. 2011, Article ID 352341 (2011)

10. Liu, Y, Nieto, JJ, Otero-Zarraquinos, O: Existence results for a coupled system of nonlinear singular fractional differential equations with impulse effects. Math. Probl. Eng. 2013, Article ID 498781 (2013)

11. Wei, X, Lu, X: The periodic solutions of the compound singular fractional differential system with delay. Int. J. Differ. Equ. 2010, Article ID 509286 (2010)

12. Zhou, WX, Chu, YD, Baleanu, D: Uniqueness and existence of positive solutions for a multi-point boundary value problem of singular fractional differential equations. Adv. Differ. Equ. 2013, 114 (2013)

13. Baleanu, D, Agarwal, RP, Mohammadi, H, Rezapour, S: Some existence results for a nonlinear fractional differential equation on partially ordered Banach spaces. Bound. Value Probl. 2013, 112 (2013)

14. Baleanu, D, Bhrawy, AH, Taha, TM: Two efficient generalized Laguerre spectral algorithms for fractional initial value problems. Abstr. Appl. Anal. 2013, Article ID 546502 (2013)

15. Baleanu, D, Diethelm, K, Scalas, E, Trujillo, JJ: Fractional Calculus Models and Numerical Methods. Series on Complexity, Nonlinearity and Chaos. World Scientific, Singapore (2012)

16. Bhrawy, AH, Alghamdi, MA: A shifted Jacobi-Gauss-Lobatto collocation method for solving nonlinear fractional Langevin equation involving two fractional orders in different intervals. Bound. Value Probl. 2012, 62 (2012)

17. Bhrawy, AH, Alofi, AS: The operational matrix of fractional integration for shifted Chebyshev polynomials. Appl. Math. Lett. 26, 25-31 (2013)

18. Bhrawy, AH, Al-Shomrani, MM: A shifted Legendre spectral method for fractional-order multi-point boundary value problems. Adv. Differ. Equ. 2012, 8 (2012)

19. Bhrawy, AH, Tharwata, MM, Yildirim, A: A new formula for fractional integrals of Chebyshev polynomials: application for solving multi-term fractional differential equations. Appl. Math. Model. 37, 4245-4252 (2013)

20. Podlubny, I: Fractional Differential Equations. Mathematics in Science and Engineering, vol. 198. Academic Press, San Diego (1999)

21. Krasnoselski, MA: Positive Solutions of Operator Equations. Noordhoff, Groningen (1964)

10.1186/1687-1847-2013-359

Cite this article as: Baleanu et al.: The existence of solutions for a nonlinear mixed problem of singular fractional

differential equations. Advances in Difference Equations 2013, 2013:359

\section{Submit your manuscript to a SpringerOpen ${ }^{\circ}$ journal and benefit from:}

- Convenient online submission

- Rigorous peer review

Immediate publication on acceptance

- Open access: articles freely available online

- High visibility within the field

- Retaining the copyright to your article 